\title{
Belphégor
}

Littérature populaire et culture médiatique

$17 \mid 2019$

Mutations des légitimités dans les productions culturelles contemporaines

\section{La bande dessinée comme forme académique}

Réflexions sur Le Déploiement de Nick Sousanis

\section{Maxime Hureau}

\section{(2) OpenEdition}

\section{Journals}

Electronic version

URL: https://journals.openedition.org/belphegor/1882

DOI: 10.4000/belphegor.1882

ISSN: 1499-7185

Publisher

LPCM

Electronic reference

Maxime Hureau, "LA Bande dessinée COMme forme aCadémique", Belphégor [Online], 17 | 2019,

Online since 16 April 2019, connection on 25 August 2021. URL: http://journals.openedition.org/

belphegor/1882 ; DOI: https://doi.org/10.4000/belphegor.1882

This text was automatically generated on 25 August 2021.

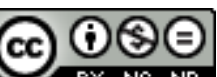

Belphégor est mis à disposition selon les termes de la Licence Creative Commons Attribution - Pas d'Utilisation Commerciale - Pas de Modification 4.0 International. 


\title{
La bande dessinée comme forme académique
}

\author{
Réflexions sur Le Déploiement de Nick Sousanis
}

Maxime Hureau

1 Depuis les années 1970 en France, les rapports entre bande dessinée et savoir s'actualisent au moins de trois manières concomitantes:

2 1) Des pédagogues la perçoivent comme un outil potentiel. Des réflexions sont menées notamment à partir de la publication de l'ouvrage d'Antoine Roux, La bande dessinée peut être éducative ${ }^{1}$.

3 2) L'Université se saisit de la bande dessinée pour en faire un objet d'investigation. Cette époque est marquée en particulier par des études sémiologiques comme l'illustrent des travaux de Pierre Fresnault-Deruelle dans la revue Communications ${ }^{2}$.

4 3) Certaines maisons d'édition l'utilisent à des fins de vulgarisation. Larousse a par exemple publié une «Histoire de France en bande dessinée » entre 1976 et 1978, en 24 tomes.

5 Ces phénomènes ${ }^{3}$ ne sont donc pas nouveaux, s'enracinent parfois même dans des pratiques bien antérieures aux années 1970, et surtout, perdurent. De fait, ils structurent historiquement la légitimité ou la reconnaissance symbolique croissante mais discutée de la bande dessinée ${ }^{4}$.

6 À l'entrecroisement de ces trois tendances, un cas particulier est apparu en France en 1975 : une première thèse réalisée en bande dessinée (fig. 1). Soutenue par Serge Tisseron à Lyon pour l'obtention du grade de docteur en médecine, elle visait à promouvoir les formes de la bande dessinée pour transmettre des connaissances sur l'histoire de la psychiatrie ${ }^{5}$. 

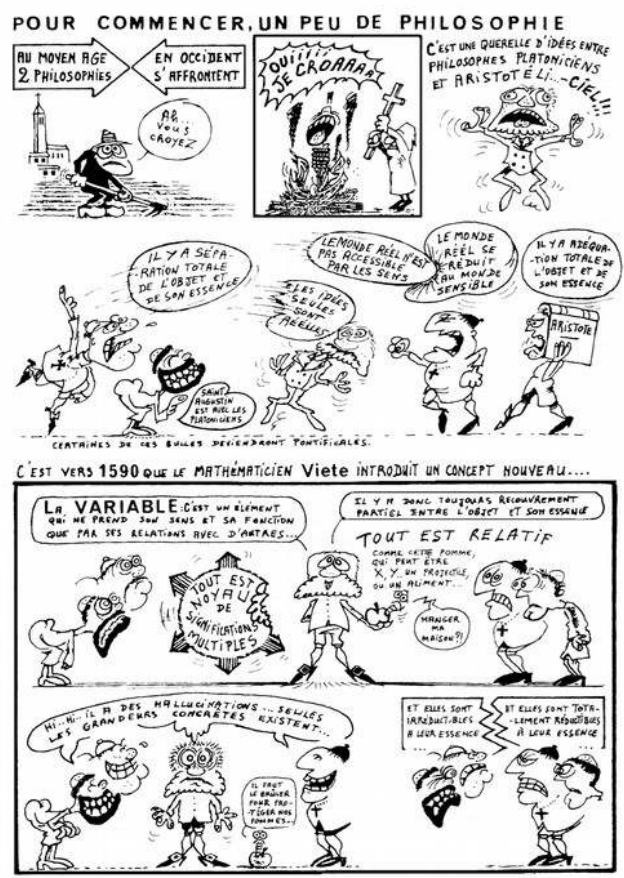

Figure 1 : Serge Tisseron, Contribution à l'utilisation de la bande dessinée comme instrument pédagogique : une tentative graphique sur l'histoire de la psychiatrie, p. 4.

7 Devenu psychanalyste, l'auteur de cette thèse a écrit en 2009 un article au titre explicite : «Passer sa thèse en bandes dessinées. C'est possible et c'est nécessaire ! ». Il revient sur sa démarche, en souhaitant ainsi « [communiquer] à de nombreux étudiants le désir de passer leurs diplômes sous la forme de bandes dessinées, voire de films ou d'animations... $»^{6}$ Différentes questions naissent alors d'une telle invitation visant à dépasser le seul langage écrit ou verbal, d'autant plus qu'une thèse en bande dessinée a été traduite en France récemment. Il s'agit de Unflatenning, soutenue par Nick Sousanis en sciences de l'éducation à l'Université de Columbia ${ }^{7}$ (New York) en 2014, et éditée à peine deux ans plus tard en France sous le titre Le Déploiement ${ }^{8}$. Ce livre apparaît comme un exemple particulièrement pertinent pour réfléchir sur la bande dessinée comme forme académique, car il est à la fois ambitieux par sa longueur (132 planches) et connaît une réception (relativement) large, en défendant au moyen de la bande dessinée la nécessité d'utiliser la bande dessinée pour des travaux universitaires.

À partir d'une telle affirmation et de cet exemple récent, on peut en effet s'interroger : en quoi une thèse dessinée repousserait-elle le primat de l'écrit pour renouveler les discours du savoir ? Comment la bande dessinée peut-elle intervenir ? Quelles conceptions de la recherche (scientifique) et de la bande dessinée sont alors défendues? Finalement, ce qui pourrait sembler un tournant paradigmatique est-il véritablement "nécessaire" comme le clame Serge Tisseron en 2009 après son expérience pionnière de 1975 ?

9 Cet éloge du « langage » de la bande dessinée en vue de la rédaction d'une production académique et/ou scientifique ne doit pas négliger les portées théoriques du discours effectivement supporté. D'un côté, les commentaires positifs concernant une telle expérience traduiraient une légitimation accrue de la bande dessinée, mais sans 
relation au contenu. Cette légitimation serait alors ambiguë, car d'un autre côté la bande dessinée, comprise comme langage, ne serait pas soumise aux mêmes normes académiques et aux attentes scientifiques les plus rigoureuses des disciplines concernées. Ce choix formel si marqué et si commenté se placerait plutôt au sein d'un cadre épistémologique postmoderne, accompagné d'une perspective politique libérale.

Pour apprécier ces hypothèses, nous remettons dans un premier temps en contexte l'œuvre de Nick Sousanis en nous demandant si nous allons vers une multiplication de thèses en bande dessinée. Ensuite, nous examinons les affirmations et les propositions formelles qui parcourent Le Déploiement, pour dégager enfin les cadres épistémologique et politique sous-jacents.

\section{I) Vers la légitimation et la multiplication de la bande dessinée académique}

\section{a) Développement de la bande dessinée universitaire}

11 Après l'expérience de Serge Tisseron citée précédemment, il n'y a pas eu de successeurs immédiats (du moins, à notre connaissance) pour formuler des discours académiques et/ou scientifiques en bande dessinée.

12 Aux États-Unis, des dessinateurs non-universitaires se sont attachés à expliquer ce qu'était la bande dessinée. Parmi eux, Scott Mccloud a réalisé, à la suite du travail pédagogique du dessinateur Will Eisner, toute une bande dessinée sur son art, intitulée L'art invisible . Parue en 1993 aux États-Unis et traduite en 1999 en France, elle a eu un fort retentissement dans les deux pays. Aujourd'hui encore, elle est citée et perçue comme une référence théorique pertinente dans de nombreux écrits universitaires sur la bande dessinée. Cet ouvrage a ouvert la brèche, probablement plus que l'exemple de $\mathrm{S}$. Tisseron, à la bande dessinée comme forme universitaire ; $\mathrm{N}$. Sousanis cite d'ailleurs le livre de $\mathrm{S}$. McCloud dans Le Déploiement ${ }^{10}$. L'idée a été reprise et s'est propagée ces dernières années à l'Université.

13 Ainsi, après un numéro de la revue Visual Art Research tout en bande dessinée (Université d'Illinois) en $2012^{11}$, un autre numéro entier de la revue Digital Humanities Quarterly, intitulé "Comics as Scholarship »" ${ }^{12}$ a été mis en ligne aux États-Unis en 2015. Dans l'appel à participations qui a lui-même été dessiné, on retrouve précisément, entre autres, une citation graphique de la couverture de l'ouvrage de S. McCloud. (fig. 2) 


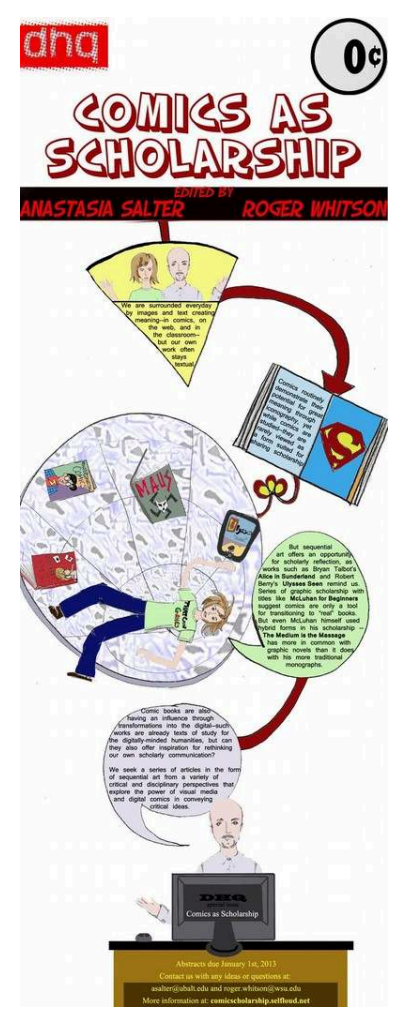

Figure 2 : Anastasia Salter et Roger Whitson, Appel à communications « Comics as Scholarship ».

14 Il existe une autre revue, également en ligne, qui se consacre uniquement à des articles en bande dessinée et qui évoque, cette fois encore, le travail de S. McCloud ${ }^{13}$. Intitulé «Sequentials » ${ }^{14}$ (Université de Floride), son premier numéro est paru en 2017.

En France, des universitaires tentent aussi depuis peu d'importer l'idée de productions académiques sous forme de bande dessinée, à l'instar de Pierre Nocerino. Doctorant en sociologie à l'EHESS, il a déjà publié plusieurs articles en bande dessinée, dont un premier à partir de son mémoire de Master 2, intitulé «Comprendre la gestion de l'autonomie en EHPAD $»^{15}$ avec Léa Mazé, puis dans une démarche méta-discursive, « Ce que la bande dessinée nous apprend de l'écriture sociologique ${ }^{16}$ en 2016 (fig. 3), reprenant la notion de s sciences sociales graphiques $\gg{ }^{17}$ proposée par l'historien Yvan Jablonka en 2014. Nicolas Labarre, qui est chercheur à Bordeaux-III et travaille justement sur l'histoire culturelle des États-Unis, a aussi publié en ligne des articles d'analyse d'objets culturels en bande dessinée, comme sur Shaun of the Dead ${ }^{18}$ ou pour le premier numéro de la revue états-unienne Sequentials ${ }^{19}$. Surtout, il a initié un appel à communication avec Marie Gloris Bardiaux-Vaïente afin de réaliser une revue de «comics universitaire ${ }^{20}$, pour un numéro hors-série d'Essais (revue de l'école doctorale Montaigne - Humanités de l'Université de Bordeaux) en septembre $2016^{21}$. Il a également contribué à un autre appel, pour la revue en ligne internationale The Comics Grid: Journal of Comics Scholarship, en mars 201722. Nicolas Labarre a aussi consacré la fin de son mémoire d'Habilitation à Diriger des Recherches au sujet de sa pratique de la bande dessinée à l'Universitée 23 . 


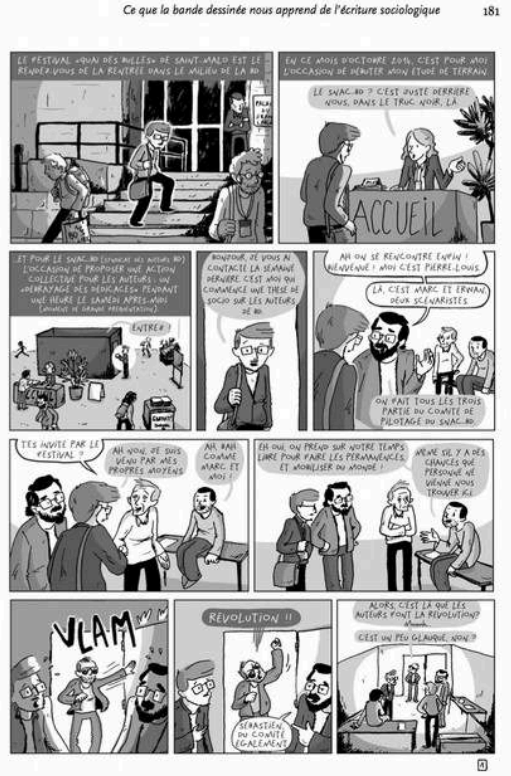

Figure 3 : Pierre Nocerino, «Ce que la bande dessinée nous apprend de l'écriture sociologique », planche introductive.

16 Ces différents exemples montrent que, bien que restant très minoritaire à l'Université, la bande dessinée académique apparait s'affirmer depuis quelques années, en bénéficiant notamment d'une circulation transnationale (des États-Unis à l'Europe, dont la France) et disciplinaire (dont l'histoire, la sociologie, les sciences de l'éducation, les études culturelles), malgré les difficultés inhérentes à ce type de projet ${ }^{24}$.

\section{b) La réception de la thèse de $\mathrm{N}$. Sousanis : la légitimation d'une forme}

Même si d'autres auteurs avaient déjà réalisé des bandes dessinées universitaires avant la publication de sa thèse, la réception du travail de Nick Sousanis, aux États-Unis comme en France, témoigne de ce développement.

18 En effet, cette thèse a été publiée quelques mois après sa soutenance aux prestigieuses Harvard University Press. N. Sousanis lui-même a été invité à dessiner un article (avec Richard Monastersky) sur la Cop21 dans la célèbre revue Nature ${ }^{25}$. L'auteur avait par ailleurs déjà participé à différentes revues en pré-publiant sa thèse ${ }^{26}$. L'ouvrage a quant à lui été récompensé par de nombreux prix, dont le Lynd Ward Prize en 2016 (prix du meilleur roman graphique paru aux États-Unis, délivré par le Centre du Livre de Pennsylvanie et l'Université de Pennsylvanie) et le $\operatorname{PROSE}^{27}$ (prix de l'Association des Éditeurs Américains, qui récompense des productions académiques, ici dans la catégorie Humanités, spécialité Media \& Cultural Studies).

19 Tout cela concrétise à la fois l'intérêt du champ de la bande dessinée et celui de la recherche universitaire, signalant ainsi une reconnaissance croissante de la bande dessinée, acceptée en tant que forme académique. 
20 En France, la thèse de N. Sousanis est traduite ${ }^{28}$ chez Actes Sud - l'An 2 dès 2016, au moment où la bande dessinée non-fictionnelle connaît une certaine prospérité. Si la maison d'édition est moins prestigieuse sur le plan universitaire, elle est tout de même dirigée par Thierry Groensteen tandis que le livre est préfacé par Thierry Smolderen. Tous deux sont des spécialistes reconnus de la bande dessinée sur le plan international avec en outre un rôle institutionnel : l'un étant, entre autres choses, l'ancien directeur du Musée de la bande dessinée (1993 à 2001), toujours commissaire d'exposition et rédacteur en chef de la revue de la Cité Internationale de la bande dessinée, l'autre est notamment l'actuel directeur du Master «bande dessinée » à l'École Européenne Supérieure de l'Image d'Angoulême.

21 De plus, les discours portés sur le livre sont élogieux, à commencer par la préface de T. Smolderen, qui le désigne effectivement comme "une brèche assez inouïe dans le monde de la recherche universitaire ». Il ajoute, après avoir résumé en une phrase la position de l'auteur :

Mais ce qui frappe le lecteur, c'est la manière dont le discours est simultanément relayé - et considérablement enrichi - par une forme de pensée en images capable de s'emparer des concepts les plus abstraits et de les déplier dans un espace beaucoup plus incarné et inventif que celui du texte [...]. ${ }^{29}$

La préface met en avant la forme particulière de cette thèse en abordant "l'essai en bande dessinée $»^{30}$ bien plus que son propos ou ses arguments, pour en défendre ou du moins en introduire la publication ${ }^{31}$.

23 Finalement, tant du point de vue des pratiques qui se développent que des discours qui les accompagnent ${ }^{32}$, différents éléments nous permettent d'observer une légitimité accrue de la bande dessinée face au savoir. Elle apparaît effectivement, pour un certain nombre de chercheurs, comme un langage possible et pertinent pour mener à bien une recherche universitaire. Toutefois, si cette reconnaissance passe d'abord par un éloge de ce «langage », elle semble négliger le contenu effectif qui est véhiculé et qui justifie sa pratique. En quoi N. Sousanis développe-t-il une alternative à ce qui serait la vision dominante de l'éducation lorsqu'il vante les qualités de la bande dessinée?

\section{II - Le Déploiement : défense et illustration d'une « science » dessinée}

$\mathrm{Au}$ travers d'un argumentaire en huit chapitres et deux interludes, l'auteur annonce qu'il veut proposer une "approche inédite " pour « découvrir les nouvelles façons de voir, ouvrir des espaces au possible et trouver des "méthodes fraîches" d'animation et d'éveil. $\rrbracket^{33}$ Cette approche passe notamment par trois points que nous résumons comme suit : d'abord par une multiplication des points de vue ${ }^{34}$; ensuite par une réflexion sur le médium utilisé, où le texte doit être conjugué au dessin ${ }^{35}$; et, enfin, par un engagement du corps qui passe par le dessin, car percevoir et penser iraient de pair ${ }^{36}$.

\section{a) La bande dessinée pour exprimer la « pensée visuelle »}

25 Afin de défendre l'idée que la bande dessinée permet d'accéder à une autre dimension, l'auteur doit montrer ce que seraient des spécificités, ou du moins des qualités, de la bande dessinée : en plus de la combinaison du texte et de l'image (p. 68-69) et l'idée de 
" séquence » conçue avec S. Mccloud (p. 71), il aborde les travaux de T. Groensteen sur le « système » de la bande dessinée (p. 72, fig. 4).

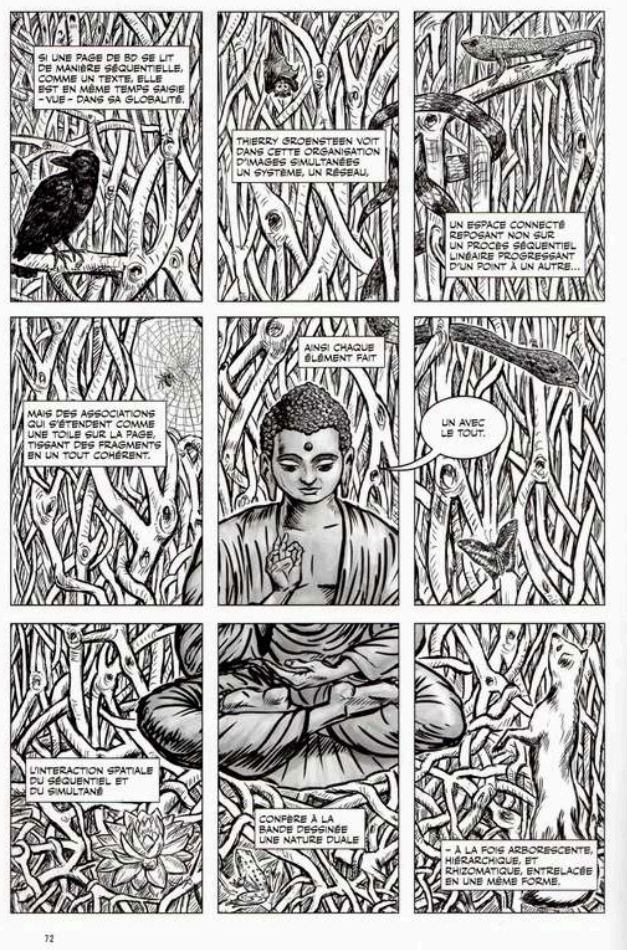

Figure 4 : Nick Sousanis, Le Déploiement, p. 72.

À ce titre, la double-page 76-77 (fig. 5 et 6) est exemplaire du Déploiement, avec un résumé didactique, grâce au cartouche contenant les termes «au cours de ce chapitre ", et plus largement l'abondance du texte qui se superpose aux dessins mis en séquence. À l'image de l'essentiel de l'ouvrage, ce texte est une voix-off placée au sein de cartouches qui découpent certaines phrases. Ces cartouches s'inscrivent sur les dessins, parfois très significativement, comme le segment «relation des composants » qui se superpose au blanc intericonique pour mieux le signaler. Conformément à l'idée de déploiement parcourant sa thèse, l'auteur, par la mise en perspective de planches construisant un parallélépipède, souligne l'importance de la spatialité pour la bande dessinée : il multiplie les images afin d'affirmer que « la BD peut rendre la manière non plate dont la pensée se déploie ». 


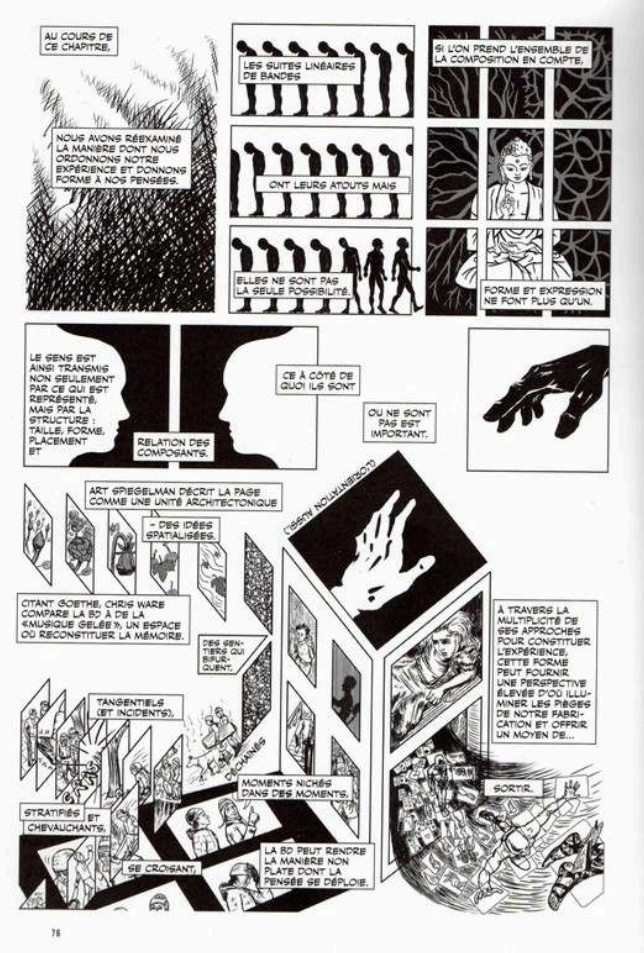

Figure 5 : Nick Sousanis, Le Déploiement, p. 76.

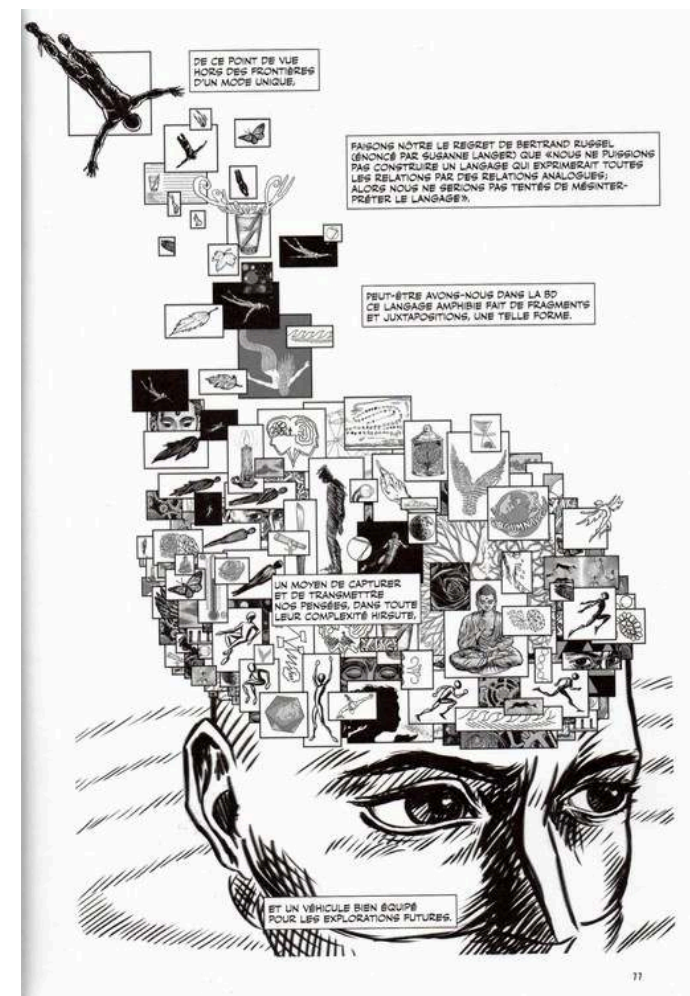

Figure 6 : Nick Sousanis, Le Déploiement, p. 77.

De plus, l'auteur reprend des dessins aperçus dans des pages précédentes. Par exemple, le Bouddha en haut sur la page de gauche (p. 76) est une citation, à l'échelle d'une case (cf. case 1), de la planche 72. Cette planche (p. 72) vise à exacerber à la fois une tension 
et une complémentarité entre le tout (la planche) et ses parties (la case). Or, elle est simplifiée page 76 en conservant seulement son gaufrier, puis devient véritablement une case sur la planche suivante (p. 77), d'abord par la citation du détail éloquent des yeux de la figure, puis par la citation de la figure entière dans une seule et même case. Cette triple reprise illustre, comme le texte de la planche 72 le mentionne mais à une échelle plus large (c'est-à-dire non plus celle de la planche, mais du livre), un aspect dual de la bande dessinée, entre saisie du sens par la séquence (lecture "linéaire ») et saisie par l'aspect global (lecture par "associations») en attirant l'attention sur la perception visuelle. Les autocitations permettent également d'évoquer l'importance des images pour la mémorisation de concepts, tout en la sollicitant. De fait, ces pages, qui ont vocation à former un résumé, présentent la bande dessinée comme « un espace où reconstituer la mémoire ».

Par un effet qui peut évoquer le «tressage $»^{37}, \mathrm{~N}$. Sousanis utilise en tout cas largement, dans tout Le Déploiement, le «modèle éclaté » du « réseau » ${ }^{38}$ qui présiderait selon $\mathrm{T}$. Groensteen au système de la bande dessinée. Dans un même ordre d'idée, le visage principal au bas de la page de droite (p. 77) provient de la page 63 pour réactiver l'idée de "langage amphibie $"^{39}$ que serait, selon N. Sousanis, la bande dessinée. L'auteur opère des variations sur ce qui constituerait donc le «langage » de la bande dessinée (case, séquence, planche, etc. d'un côté, texte et image de l'autre) afin de rendre compte de son intérêt cognitif. Tel semble être l'enjeu du Déploiement: exposer le dispositif et illustrer ses modalités, car elles conduiraient à penser différemment en permettant de développer la « pensée visuelle ».

\section{b) Une thèse réflexive : dessiner sur la bande dessinée}

L'exhibition de la réflexivité dont fait preuve N. Sousanis corrobore l'idée selon laquelle Le Déploiement relèverait de cette pensée visuelle en acte, en nouant dessin, perception et «pensée ».

Pour ce faire, l'auteur se représente en dessinateur-pensant (fig. 7, p. 89) à la fin du chapitre quatre qui vise à affirmer que la " pensée » dépend aussi de notre « expérience physique du monde $\aleph^{40}$. Pour synthétiser cette idée, N. Sousanis reprend en fin de chapitre "Le Penseur» de Rodin. Cette sculpture redessinée constitue une image archétypale de la pensée, soutenue par un code classique de la bande dessinée : les bulles de pensée qui émergent (en haut à gauche) d'une tête cloisonnée dans une case isolée sur la page. 


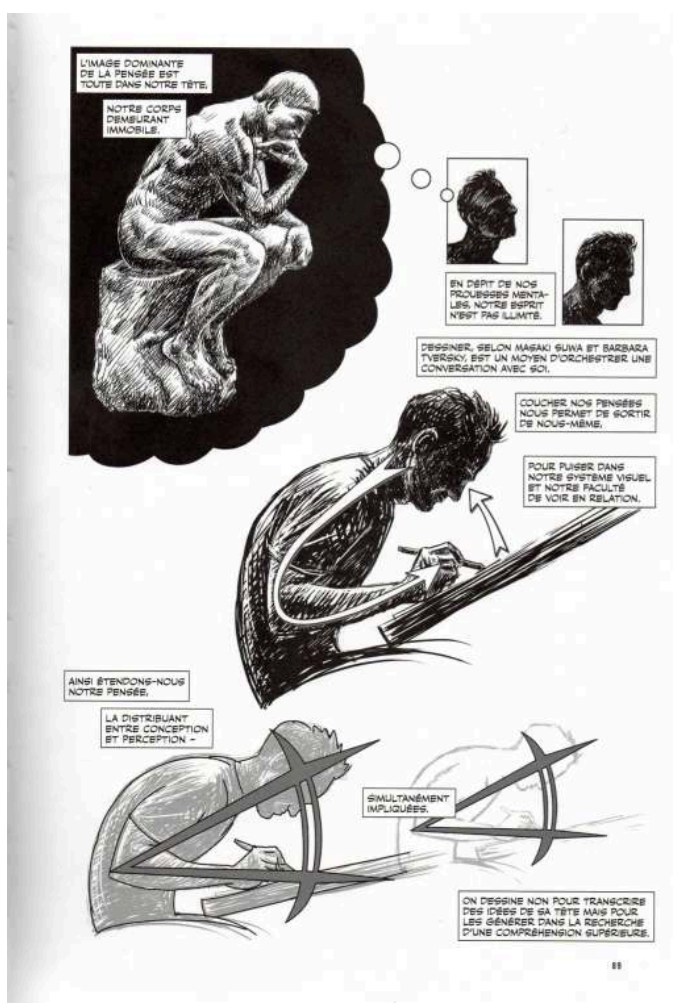

Figure 7 : Nick Sousanis, Le Déploiement, p. 89. position du corps forme un compas ou l'ouverture d'un œil ${ }^{41}$. Bien que pris aussi en charge par le texte, ce procédé permet de lier par le dessin l'activité de « perception » à celle de « conception ». Sur le premier portrait de l'auteur à sa table - qui est une figure par ailleurs relativement courante en bande dessinée - les flèches soulignent cette correspondance. L'auteur conclut la planche par cette idée sur le dessin en dévoilant ce qui est à la fois la méthode et l'objet de sa thèse : «On dessine non pour transcrire des idées de sa tête mais pour les générer dans la recherche d'une compréhension supérieure $\aleph^{42}$. Dit autrement, les théories qui animent $\mathrm{N}$. Sousanis sont soutenues d'abord par l'exemple qu'il produit lui-même; la forme justifie le propos, et inversement.

\section{c) Une thèse narrative qui valorise l'imagination}

Malgré son statut académique, de nombreux aspects narratifs voire fictionnels servent aussi à appuyer l'argumentation de l'auteur, qui inclut ainsi diverses références à des œuvres de fiction et deux interludes.

Le premier interlude reprend le roman Flatland d'Edwin Abbott Abbott (paru en 1884), dans lequel le narrateur est un carré, qui rencontre d'autres formes géométriques et voyage de dimension en dimension (il passe par exemple de la première dimension, plate, à la deuxième dimension d'où il vient, etc.). Les personnages géométriques qui peuplent ce Flatland sont repris tout au long de l'ouvrage (par exemple p. 85) alors que le roman est explicitement cité (textuellement) six fois, dont une fois avec le possessif : " nos flatlandais ». Le titre original de la thèse (Unflatenning) offre une place centrale à cette référence. Le deuxième interlude relève aussi du conte. L'adresse directe au 
lecteur l'invite à imaginer un pantin, qui n'est pas sans évoquer une variation sur Pinocchio. Enfin, les nombreuses citations qui parcourent Le Déploiement proviennent parfois d'œuvres de fiction, dont parmi elles, certes Flatland, mais également Le Magicien d'Oz ou Ulysse de James Joyce, tandis que des références sont empruntées aux comics de super-héros, avec Batman, ainsi qu'à la mythologie, avec Persée et Hermès.

L'introduction de la fiction et l'usage de techniques narratives sont relativement fréquents dans la vulgarisation scientifique ${ }^{43}$. Cependant, dans cette thèse, ces interludes permettent aussi de soutenir la valorisation de l'imagination opérée par $\mathrm{N}$. Sousanis au cinquième chapitre. Selon lui, «l'imagination » permettrait de dépasser notre propre point de vue et de «ressentir le rapport de l'autre au savoir ${ }^{44}$; l'imagination serait alors la cinquième dimension, directement liée aux deux précédentes, à savoir la multiplication des points de vue et le dessin. À nouveau, la démarche légitime la thèse en plaçant en miroir la théorie défendue et la manière de l'inscrire.

\section{d) Une thèse critique de l'éducation, de la technique et de la raison}

35 Ces valorisations de la réflexivité par le dessin, des spécificités de la bande dessinée, de la narration et de l'imagination, s'accompagnent d'un discours critique qui a motivé les «nouvelles" propositions présentées précédemment. Ces critiques portent sur la technique, sur l'éducation, voire sur la raison. N. Sousanis plaide pour la bande dessinée afin d'avoir accès à d'autres dimensions précisément parce qu'il considère que l'éducation contemporaine et ses fondements contribuent à la "planitude $»^{45}$ qu'il $^{\prime}$ dénonce.

Le premier chapitre de la thèse décrie, en s'appuyant sur Herbert Marcuse qui parle de "schéma de pensée et de comportement unidimensionnels", la standardisation de l'être humain, formaté sur des lignes de production, dans des usines, les corps recevant un traitement graphique unique et se limitant à des silhouettes, en référence ici à la science-fiction de Metropolis de Fritz Lang ${ }^{46}$. Une scène évoque directement un contexte d'enseignement (p. 20): un visage diffuse des images (des cases vides ou noires) traversant le cerveau de silhouettes identiques, momifiées et courbées sur un bureau pour prendre des notes.

N. Sousanis désigne aussi des "ornières" dans son chapitre 6 ; en fait, ce sont les structures et dispositifs techniques (par exemple dès p. 22, ou p. 119) que nous avons créés pour appréhender (observer, mesurer) le monde qui nous entoure. Selon lui, ces dispositifs ont aussi, à terme, un effet néfaste sur notre perception.

À l'image du passage du ticket de métro (p. 121) que nous ferions machinalement, nous serions pris dans nos habitudes et compréhensions du monde façonnées par une technique qui n'était pas censée s'appliquer à cadrer ainsi l'être humain.

Finalement, par ces deux premières critiques, la raison est mise en cause, au sens de « raison instrumentale » comme pourraient le dire des tenants de l'École de Francfort, tels que Theodor W. Adorno et Max Horkheimer, inspirant notamment le premier chapitre et cités en notes ${ }^{47}$, ou $\mathrm{H}$. Marcuse déjà évoqué. Cet élément est peut-être moins net que les deux premiers mais s'exprime par exemple grâce à une citation d'Italo Calvino qui intervient dès le premier chapitre : 
[...] Je veux dire qu'il me faut changer d'approche, qu'il me faut considérer le monde avec une autre optique, une autre logique, d'autres moyens de connaissance et de contrôle. ${ }^{48}$

\section{III - Limites épistémologiques et conclusions politiques du Déploiement}

Un regard global sur la forme de la thèse de $\mathrm{N}$. Sousanis suggère que l'auteur respecte les attentes d'un travail universitaire. Il cite par exemple d'autres travaux tout au long de sa démonstration, aboutissant à onze pages de bibliographie, même si les références sont éclectiques et parfois anciennes ${ }^{51}$.

En revanche, rares sont les définitions des concepts, et certains éléments qu'il critique ne sont pas présentés voire verbalisés. Ainsi, N. Sousanis ne nous dit pas ce qu'est la « pensée » pour lui (est-ce l'activité cérébrale continue ? l'effort cognitif pour une tâche définie? la démarche rationnelle de manière générale ? la philosophie ?) ni la « compréhension supérieure » issue de la réflexivité par exemple. De manière générale dans Le Déploiement, il nous semble que la relative nouveauté de la "bande dessinée académique" a tout de même tendance à occulter une démarche scientifique au profit d'une forme plus métaphorique et littéraire, voire ésotérique.

\section{a) La métaphore omniprésente : ambiguitté d'une figure}

D'ailleurs, et N. Sousanis le reconnaît, l'ensemble de la thèse qu'il effectue en bande dessinée est une métaphore : «[...] à travers sa forme même elle soutient un argument métaphorique pour l'importance de la pensée visuelle dans l'enseignement et l'apprentissage $»^{52}$. Parfois mobilisée par le texte, elle peut endosser en même temps une dimension concrète en étant redoublée par le dessin ; elle peut aussi être filée par le seul dessin ou produire des doubles sens. Les différentes potentialités de la métaphore conduisent parfois à une indétermination du sens qui peut être problématique.

Par exemple, la perspective est une métaphore qui se transforme en argument pour présenter la multiplication de points de vue (par exemple p. 53) et l'approfondissement de la compréhension dans Le Déploiement. Certes, on peut interpréter cette idée telle

Belphégor, 17 | 2019 
que N. Sousanis l'avance dans un article consacré à la réalisation de sa propre thèse, c'est-à-dire comme une défense de l'interdisciplinarité :

Je suggère des pistes pour dépasser la planitude en engageant une discussion sur l'interdisciplinarité par la métaphore de la perspective - [...] (à nouveau, je n'utilise jamais de termes tels que "discipline" ou "interdisciplinarité"). ${ }^{53}$

Cela dit, il nous semble que cette planche, mise en réseau, par exemple avec la page 49 (fig. 8) pourrait être lue aussi comme une adhésion au relativisme, d'autant plus que l'auteur souligne à nouveau ne pas désigner ce dont il déclare a posteriori discuter. Cette défense du relativisme passerait par la multiplication non plus des points de vue disciplinaires mais individuels. Le foisonnement des yeux, au travers d'une mise en page en "rhizome" - la métaphore deleuzienne est citée par l'auteur sur la même planche - suggère ainsi «les manières de voir propres à chacun de nous ». Mais l'individualisation ne serait-elle pas, à son tour, une simple métaphore? En tout cas, ce type de formules renvoyant le problème à un ordre subjectif revient à plusieurs reprises dans l'ouvrage ${ }^{54}$. S'y ajoute parfois l'introduction de la subjectivité dans le discours auctorial, donnant une tonalité essayiste à cette thèse $\mathrm{e}^{55}$.

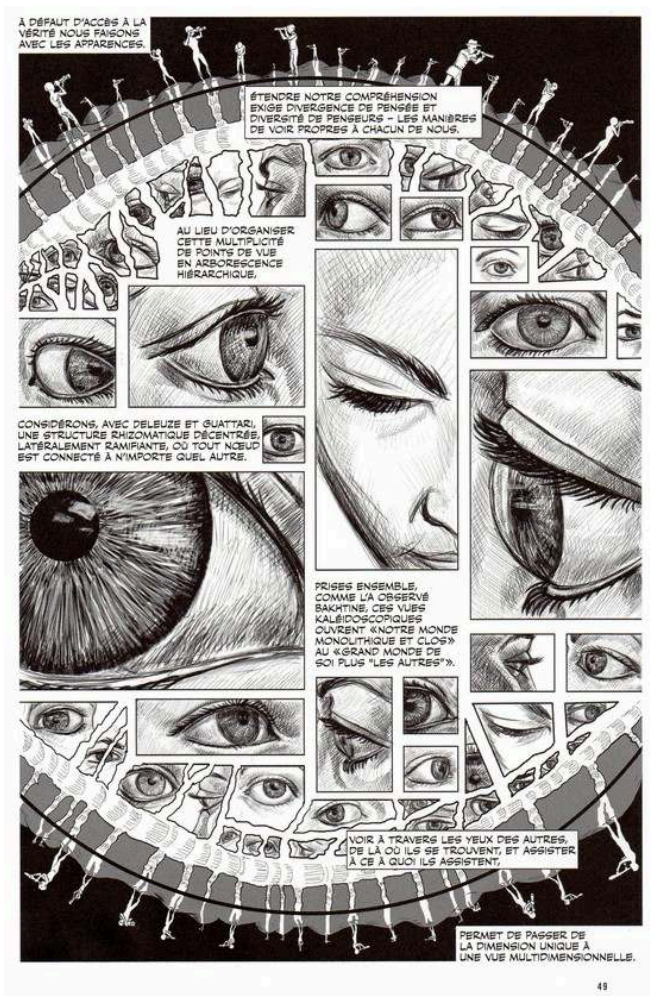

Figure 8 : Nick Sousanis, Le Déploiement, p. 49.

Dans Prodiges et vertiges de l'analogie, le philosophe Jacques Bouveresse dénonce les excès de métaphore et épingle en particulier la prétention démonstrative d'une telle pratique ${ }^{56}$, notamment au sein de textes issus de ce qu'on a appelé le post-modernisme, ou la French Theory, qui défendaient justement un certain relativisme cognitif. D'après François Cusset qui a travaillé sur la réception de cette French Theory aux États-Unis, celle-ci serait « mystérieusement intransitive, sans autre objet que son énigme : elle est avant tout discours sur elle-même, et sur les conditions de sa production - donc sur l'université. $»^{57} \mathrm{Il}$ ajoute qu'elle interrogerait « sans répit sa propre légitimité $»^{58}$, ce qui n'est pas sans faire écho à la réflexivité à l'œuvre dans Le Déploiement. En d'autres 
termes, N. Sousanis semble produire ce genre de "Théorie », encourageant encore l'interprétation de la métaphore de la perspective comme illustration du relativisme.

\section{b) Du relativisme à l'abus conceptuel}

48 Tout en permettant par ailleurs d'introduire l'idée du relativisme cognitif, le point de départ du voyage qu'entreprend $\mathrm{N}$. Sousanis - car il utilise aussi cette métaphore relève de ce qu'Alan Sokal et Jean Bricmont ont appelé «impostures intellectuelles ». Ils définissent l'imposture intellectuelle comme «l'abus réitéré de concepts et de termes provenant des sciences physico-mathématiques $"^{59}$, ou le fait de détourner des théories scientifiques, de les extrapoler sans même en expliquer la présence, parfois pour le jeu de mots, en leur faisant dire ce qu'elles ne peuvent pas permettre d'affirmer.

49 À la page 41 (fig. 9), N. Sousanis déclare dans le cartouche entre les deux yeux que « la distance séparant nos yeux implique une différence entre leurs visions respectives - il n'y a donc pas de vision unique, "correcte" ». Il s'appuie alors sur une branche de la physique en avançant la notion de parallaxe. Afin d'en rendre compte, il montre une expérience (plutôt que de donner une définition précise) facilement reproductible puisque ne demandant aucun matériel autre que son propre doigt. Cette "(dé)monstration" sous le signe de l'évidence et du calembour (" cela saute aux yeux » écrit-il) sera généralisée à l'échelle astronomique ${ }^{60}$ dès la page suivante pour finalement donner une « définition de travail » du « déploiement » (soit : « engagement simultané de points d'observations multiples pour engendrer de nouvelles façons de voir $\left.»^{61}\right)$. En outre, la poursuite de la métaphore dans le chapitre, graphiquement (p. 53) ou non (p. 55), donne une place prépondérante à ce motif. Par ce biais, il commence donc à fonder et à justifier la nécessité de multiplier les points de vue et les angles d'approche de la réalité. Indépendamment de sa pertinence et de ses limites, cette idée n'est d'ailleurs finalement pas si originale. 


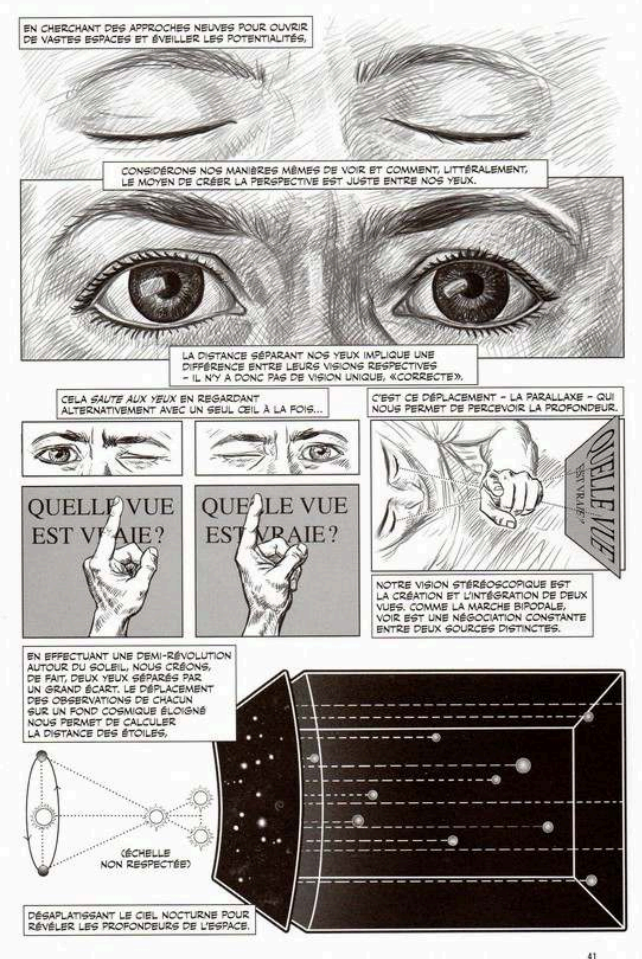

Figure 9 : Nick Sousanis, Le Déploiement, p. 41. l'auteur suggère par un nouvel effet d'individualisation qu'il n'y a pas de vérité stable nous dirions plutôt pas de fait ou de théorie ajustée - mais seulement des points de vue et des récits. Cela est plus clairement assumé avec la planche de la page 105 qui présente sur le même plan graphique (par la série de cases issues du livre des Mille et Une Nuits) le conte merveilleux et le travail d'un astronome. L'auteur ajoute ici : «Par histoires, je n'entends pas les seuls contes merveilleux, mais cette activité des plus humaines, cadrer l'expérience pour lui donner du sens. »

51 Ainsi, l'abus conceptuel par une utilisation métaphorique d'un concept scientifique sert aussi à appuyer le relativisme qui s'insinue ici par un discours individualisant. Or, ce dernier ne se rapporte pas uniquement au cadre épistémologique et à ses conséquences dans l'écriture des savoirs, mais concerne aussi le domaine politique des réflexions et propositions sur l'éducation de N. Sousanis.

\section{c) Une critique "apolitique" ou la promotion de l'individualisme libéral}

52 Pour certains et certaines, la bande dessinée pourrait apparaître comme un moyen progressiste d'aborder le problème de la connaissance et de l'enseignement, d'autant plus qu'elle bénéficie d'un effet de nouveauté à l'Université. D'ailleurs, entre autres références, la citation de $\mathrm{H}$. Marcuse affilie Le Déploiement à une tradition critique qui assumait un discours politique que nous avons pour habitude de situer à gauche (voire à l'extrême-gauche) ${ }^{62}$.

53 Cependant, en cohérence avec l'indétermination ou les ambiguïtés qu'il entretient dans sa thèse, $\mathrm{N}$. Sousanis déclare avoir eu «l'intention de créer une œuvre qui ne 
tourne pas le dos aux lecteurs avec un vocabulaire spécialisé ou politisé, mais les invite à trouver leur propre voie en connectant les informations ${ }^{63}$. Il est vrai qu'aucune position politique explicite n'est revendiquée, et que les références politisées ne le sont plus, à l'image du freudo-marxisme de l'ouvrage de H. Marcuse intitulé l'Homme unidimensionnel (1964, trad. 1968). En particulier dans les deux derniers chapitres, $\mathrm{N}$. Sousanis critique le conformisme et la résignation qu'il rassemble dans la formule « un inéluctable c'est comme ça » ${ }^{64}$. Cette dernière, écho au " There is no alternative », aurait $\mathrm{pu}$ de ce fait conduire vers une critique sociale, et rejoindre une perspective anticapitaliste et/ou antiautoritaire prêtée à $\mathrm{H}$. Marcuse. Pourtant, la critique de l'éducation de $\mathrm{N}$. Sousanis se limite à une critique de la standardisation des individus (par l'éducation) et de la soumission à des codes formels (l'écrit). D'après l'auteur, il faudrait «approcher ce qui fait de nous ce que nous sommes »65, ce qu'il illustre par le dessin de son propre pied en filant la métaphore sur les quatre pages suivantes. Sa critique se borne à une individualisation du problème en préférant un discours relatif à l'identité. Dans une certaine mesure, il s'agit de ce que Luc Boltanski et Ève Chiapello appellent la «critique artiste ", ici menée au détriment de la « critique sociale " ${ }^{66}$, et qui se focalise sur l'authenticité de l'expérience et l'individualité. Grâce à leur étude, les deux sociologues ont suggéré que cette critique artiste rencontrait un grand succès au sein des entreprises via le discours managérial qui s'était réapproprié ces valeurs en évacuant la perspective de transformation sociale qui y était exprimée originellement.

D'un autre côté, les représentations de groupes, dans Le Déploiement, sont en grande partie des représentations de foules marquant une pesanteur sociale, parfois morbide comme à la page 138 ou dans l'ensemble du premier chapitre. Même lorsque l'auteur replace, pour la première fois, l'individu dans le social à la page 143 en parlant de « tissu social » (fig. 10), ce « tissu » n'est composé que de textes en arrière-plan, relatif à l'identité du personnage représenté, sans prendre véritablement part à la démonstration générale. Pour citer un concept qu'on rattache à la culture étatsunienne, N. Sousanis reprend le mythe du self-made man pour amener sa conclusion (fig. 11, p. 154):

Trop souvent cependant, nous succombons à cette illusion que le pouvoir de déterminer qui vous êtes et le chemin devant vous ne sont pas dans vos mains mais sujets à des forces extérieures. 


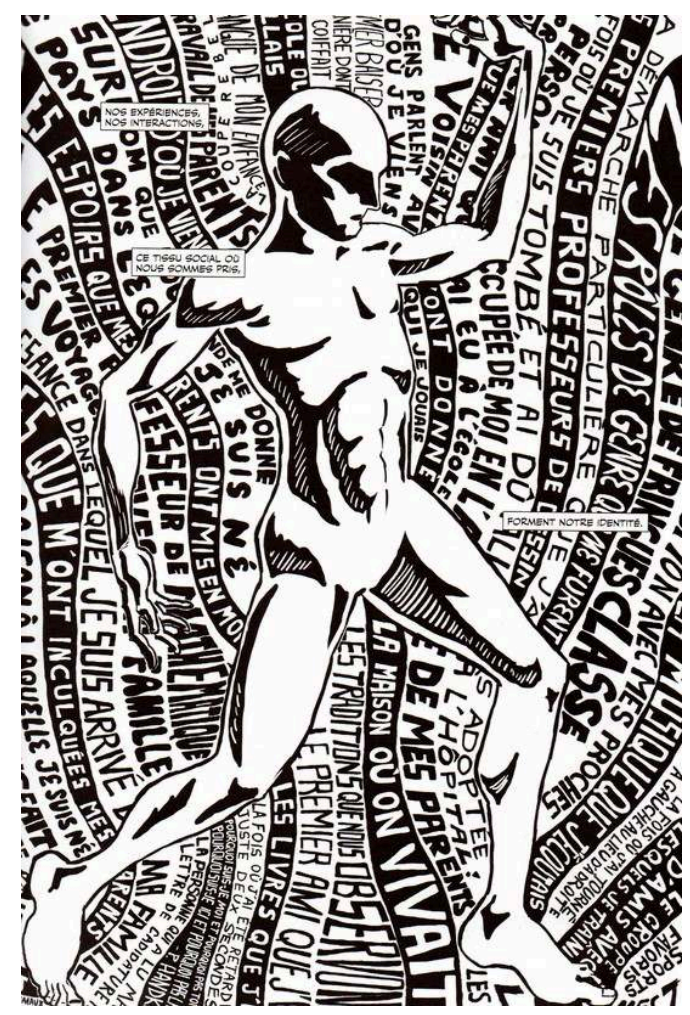

Figure 10 : Nick Sousanis, Le Déploiement, p. 143.

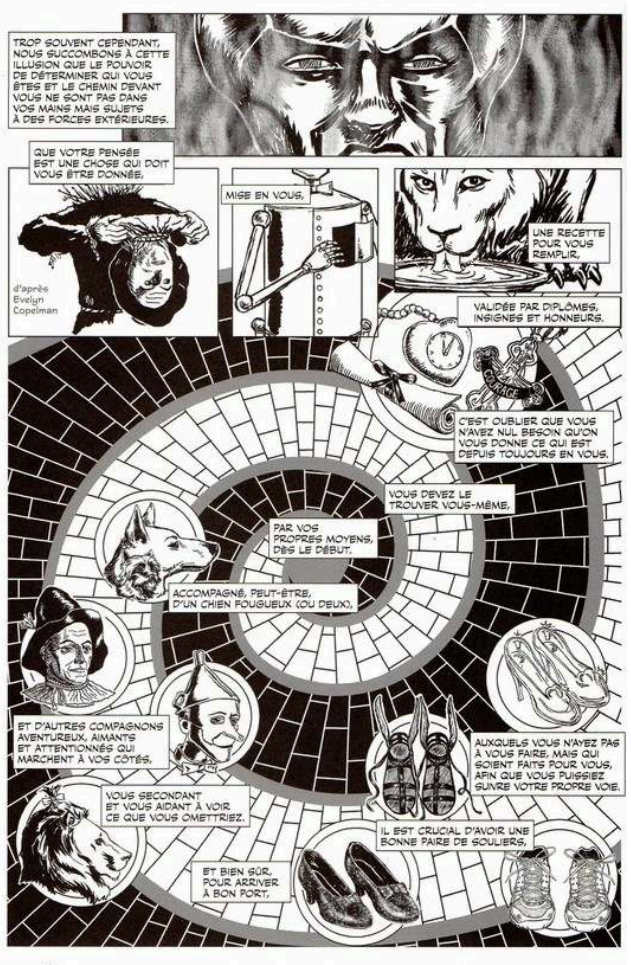

184

Figure 11 : Nick Sousanis, Le Déploiement, p. 154.

55 Cette affirmation s'inscrit en cohérence avec la norme d'internalitée ${ }^{67}$ observée par certains travaux en psychologie sociale, dont ceux de Nicole Dubois : pour expliquer ce que nous faisons et ce qui nous arrive, nous aurions une forte tendance à mobiliser des 
facteurs internes (dispositions, traits personnels, attitudes, intentions, etc. - ici : " vos mains ») plutôt que des facteurs externes (situation objective, chance, hasard, rôle d'autrui, etc. - ici: les «forces extérieures»). Or, cette norme serait acquise en particulier à l'école et socialement valorisée dans les sociétés libérales. Les propos de $\mathrm{N}$. Sousanis font plus largement la promotion de l'individualisme, que l'on peut définir selon cinq piliers, proposés par Steven Lukes en 1973 et cités par N. Dubois, et que nous résumons comme suit: a) l'individu compris comme une fin en soi; b) le développement de soi: l'objectif pour l'individu étant alors de se réaliser; c) l'autonomie : il s'agit pour l'individu de décider par lui-même, d) la vie privée : il y aurait un espace de vie hors des influences et intrusions extérieures pour l'individu ; e) l'idée que les capacités et les besoins de l'individu seraient innés ${ }^{68}$. Sur cette même page 154, au moins quatre de ces piliers apparaissent. En effet, pour prendre ses distances avec les "diplômes, insignes et honneurs ", l'auteur soutient ceci, en s'adressant au lecteur : « [...] vous n'avez nul besoin qu'on vous donne ce qui est depuis toujours en vous. [cf. c, d, e] / Vous devez le trouver vous-même, [cf. c] / par vos propres moyens dès le début. [cf. e] ${ }^{69}$ Tout ce voyage conduit à ce «que vous puissiez suivre votre propre voie. $[c f . b] \gg^{70}$ Le fait que sa thèse se termine par cette valorisation du moi relève peut-être enfin de la conception de l'individu comme fin en soi (cf. a). Malgré la concession faite à d'éventuels " compagnons aventureux " (des personnages aperçus précédemment), l'auteur soutient donc ouvertement une conclusion individualiste.

Ainsi, l'absence de discours politique explicite et la valorisation de l'individu comme perspective finale participent, de fait, d'une position libérale, alors que N. Sousanis aurait pu à l'inverse privilégier une critique sociale du système éducatif à partir d'un socle rationaliste ${ }^{71}$. En revanche, il est vrai que l'autolégitimation d'une thèse en bande dessinée n'aurait alors pas été aussi nette.

\section{Conclusion : l'esthétique triomphant sur la connaissance?}

Cette présentation du Déploiement et de quelques-uns de ses enjeux principaux avait pour but d'interroger un exemple de bande dessinée académique. Il faudrait, en approfondissant ces analyses, lui comparer d'autres productions académiques qui semblent se développer actuellement (voir supra).

Il a néanmoins paru important de montrer les limites de ce cas exemplaire dans un contexte de légitimation et d'autolégitimation. Celles-ci peuvent se résumer comme suit : prédominance de la forme dans l'argumentation, réflexivité autotélique, équivoques par la métaphore, difficulté à identifier le statut épistémologique ${ }^{72}$ des affirmations. L'ensemble rend la compréhension et la discussion de cette thèse parfois difficiles. Certes, la traduction d'une telle œuvre permet de réfléchir à la transmission de connaissances et de discuter à partir d'un cas effectif des propositions telles que celles de N. Sousanis. Cependant, comme le signale le dessinateur et neuroscientifique Matteo Farinella dans sa récente revue de questions sur la communication scientifique en bande dessinée ${ }^{73}$, d'un côté, les études sur les intérêts de la bande dessinée semblent encore limitées d'un point de vue empirique et, d'un autre, celles déjà menées le sont souvent à partir d'exemples proposés par les auteurs eux-mêmes, avec une approche qualitative. Il invite aussi à poser les questions en termes de dispositifs visuels circonscrits plus qu'en termes de «bande dessinée ». 
us largement, la thèse en bande dessinée pourrait apparaître comme un placement dans un champ artistique, et surtout, un placement dans le champ académique qui valide aujourd'hui ce type de discours. De surcroît, n'y a-t-il pas aussi des raisons économiques et stratégiques de diffusion de la recherche ${ }^{74}$ qui poussent à développer ces initiatives, dans le contexte d'un large succès de la non-fiction dessinée au sein des industries culturelles? Toujours est-il que cette thèse et sa réception marquent effectivement une grande valorisation d'un art, la bande dessinée, mais faite uniquement $\mathrm{du}$ point de vue esthétique ou formel, négligeant par exemple son inscription sociale et même son contenu. Cette valorisation lui permet d'entrer dans les sphères académiques pour rejeter un système éducatif qui limiterait nos potentialités, ici à cause du cloisonnement de la pensée par le recours au seul texte.

(linterrogation sur la forme (de transmission) de la recherche devrait a minima s'accompagner d'une réflexion générale sur le cadre social dans lequel elle s'inscrit et sur ses objectifs. Nous pourrions effectivement, pour terminer, regretter que $\mathrm{N}$. Sousanis n'aborde pas le poids des éléments externes comme l'organisation sociale (hiérarchique, inégalitaire) et son actualisation économique (le capitalisme) sur l'enseignement et la recherche scientifique. Or, ces éléments pèsent probablement bien plus que le dit "médium » de notre pensée sur les réalités tangibles dont nous faisons l'expérience quotidienne dans les universités. Cela a d'ailleurs bien été relevé en 2018 par celles et ceux qui ont notamment contesté les réformes de l'accès à l'université (le dispositif « Parcoursup » et la sélection qu'il implique), le plus souvent des étudiants et étudiantes. Mais contrairement à la thèse de $\mathrm{N}$. Sousanis, ils et elles ont été largement délégitimés voire violemment réprimés.

Les figures 4 à 11 sont présentées avec l'aimable autorisation des éditions Actes Sud l'An 2.

\section{NOTES}

1. Roux, Antoine, La bande dessinée peut être éducative, Paris, l'École, 1970.

2. Voir par exemple: Fresnault-Deruelle, Pierre, "Le verbal dans les bandes dessinées", Communications, L'analyse des images, $\mathrm{n}^{\circ} 15,1970$, p. 145-161. Fresnault-Deruelle, Pierre, « Du linéaire au tabulaire ", Communications, La bande dessinée et son discours, n² 24, 1976, p. 7-23.

3. Sur les rapports entre bande dessinée et savoir, nous pouvons renvoyer de manière nonexhaustive aux synthèses suivantes : Rouvière, Nicolas, « Enseignement (1) : enseigner avec la bande dessinée ", Dictionnaire esthétique et thématique de la bande dessinée, Neuvième Art 2.0, 2013 [en ligne]. Disponible sur : http://neuviemeart.citebd.org/spip.php?article523 Consulté le 03/09/18 ; Stefanelli, Matteo, "Un siècle de recherches sur la bande dessinée ", dans Maigret, Eric et Stefanelli, Matteo (dir.), La bande dessinée : une médiaculture, Paris, Armand Colin- Ina, 2012, p. 17-49; Groensteen, Thierry (dir.), «Les bandes dessinées de non-fiction », Neuvième art 2.0, [en ligne]. Disponible sur:http://neuviemeart.citebd.org/spip.php?rubrique159 Consulté le 03/09/18. 
4. Boltanski, Luc, "La constitution du champ de la bande dessinée ", Actes de la recherche en sciences sociales, vol. 1, n 1, 1975, p. 37-59 ; Maigret, Éric, « La reconnaissance en demi-teinte de la bande dessinée ", Réseaux, vol. 12, n 67, 1994, p. 113-140; Ahmed, Maaheen, Delneste, Stéphanie et Tilleuil, Jean-Louis (dir.), Le Statut culturel de la bande dessinée. Ambiguités et évolutions, Louvainla-Neuve, Academia - L'Harmattan, « Texte-Image », 2016.

5. Bipartite, cette thèse comprend une bande dessinée de 48 pages, et un texte d'accompagnement (p. 55-66). Tisseron, Serge, Contribution à l'utilisation de la bande dessinée comme instrument pédagogique : une tentative graphique sur l'histoire de la psychiatrie, Thèse de 3ème cycle sous la direction de Goyotat, Jean, Université Claude Bernard, Lyon I, 1975. Disponible en ligne sur : $\quad$ http://www.biusante.parisdescartes.fr/ressources/pdf/histmed-asclepiades-pdftisseron.pdf

On retrouve certaines planches éditées dans Tisseron, Serge, Histoire de la psychiatrie en bandes dessinées, Rome, Savelli, « bandes dessinées », 1977.

6. Tisseron, Serge, " Passer sa thèse en bandes dessinées. C'est possible, et c'est nécessaire ! ", Sociétés, $\mathrm{n}^{\circ}$ 106, 2009, p. 13.

7. Il s'agit, selon nous, de la section $\mathrm{CNU}$ qui correspondrait le mieux au cadre institutionnel états-unien de la thèse de N. Sousanis : « Doctorate of Education, Interdisciplinary Studies » au " Teachers College, Columbia University ».

8. Nous utiliserons l'édition française comme édition de référence : Sousanis, Nick, Le Déploiement, trad. de l'anglais par Voline, Marc, Arles, Actes Sud - L'An 2, 2016.

9. McCloud, Scott, L'art invisible (1993), trad. de l'anglais par Petitfaux, Dominique, Paris, Vertige Graphic, 1999. Le titre original souligne la visée pédagogique de l'ouvrage : Understanding Comics: The Invisible Art.

10. Sousanis, Nick, op. cit., p. 71.

11. Carpenter II, B. Stephen, et Tavin, Kevin (dir.), Visual Arts Research, « The Graphic Novel and Art Education ", vol. 38, $n^{\circ} 1,2012$. N. Sousanis a participé à ce numéro, voir infra.

12. Whitson Roger et Salter, Anastasia (dir.), Digital Humanities Quarterly, « Comics as Scholarship ", vol. 9, n 4, 2015 [en ligne]. Disponible sur : http://www.digitalhumanities.org/dhq/vol/9/4/ index.html Consulté le 03/09/18.

13. Dans la présentation de la revue en ligne, on peut lire : «Following Scott McCloud's 1993 publication Understanding Comics: The Invisible Art, as well as other visual comics scholarship from authors such as Nick Sousanis and Neil Cohn, Sequentials solicits and publishes interpretations of various academic subjects or themes drawn and explained through the comics medium.» Sequentials, « About Sequentials » [en ligne]. Disponible sur : http://www.sequentialsjournal.net/ about.html Consulté le 03/09/2018.

14. Dobrin, Sid et Manchester, Ashley (dir.), Sequentials, "Postmodernism: Visualizing a movement », vol. 1, n 1, 2017 [en ligne]. Disponible sur: http://www.sequentialsjournal.net/ Consulté le 03/09/2018.

15. Nocerino, Pierre, « Comprendre la gestion de l'autonomie en EHPAD », Lettre de l'Observatoire des Retraites, $n^{\circ} 22$, décembre 2015, p. 3-14. Sur un sujet proche avec Léa Mazé, ils ont réalisé un autre article de «sociologie dessinée » avec un avant-propos expliquant leur démarche : Mazé, Léa et Nocerino, Pierre, «Analyser l'accueil des personnes âgées en institution. De l'autonomie aux transferts de responsabilité ", ethnographiques.org, $\mathrm{n}^{\circ} 35$, Vieillir en institution, vieillesses institutionnalisées. Nouvelles populations, nouveaux lieux, nouvelles pratiques, 2017 [en ligne]. Disponible sur: http://www.ethnographiques.org/2017/Maze_Nocerino Consulté le 03/09/18. Enfin, il tient aussi un blog avec Léa Mazé sur lequel ils développent leurs réflexions entre bande dessinée, recherche et vulgarisation, sous le pseudo LMNOP : LMNOP, «Émile, on bande ? " [en ligne]. Disponible sur https://socio-bd.blogspot.com/ Consulté le 03/09/18 
16. Nocerino, Pierre, "Ce que la bande dessinée nous apprend de l'écriture sociologique ", Sociologie et sociétés, vol. 48, $\mathrm{n}^{\circ}$ 2, automne 2016, p. 169-193. Disponible en ligne sur: https:// www.erudit.org/fr/revues/socsoc/2016-v48-n2-socsoc02692/1037720ar.pdf

17. Jablonka, Yvan, "Histoire et bande dessinée ", La vie des idées, Dossier "Les formes de la recherche », 2014 [en ligne]. Disponible sur: http://www.laviedesidees.fr/Histoire-et-bandedessinee.html Consulté le 03/09/18.

18. Baillon, Jean-François et Labarre, Nicolas, « Things are Going to Change: Genre Hybridization in Shaun of the Dead", Angles: French Perspectives on the Anglophone World, «Brevity is the soul of wit ", 2015 [En ligne]. Disponible sur: http://angles.saesfrance.org/index.php?id=96 Consulté le 03/09/18.

19. Labarre, Nicolas, "The Popular as Postmodern in Michael Moorcock's Early Work», Sequentials, «Postmodernism : Visualizing a movement », vol. 1, $\mathrm{n}^{\circ} 1,2017$ [en ligne]. Disponible sur : http://www.sequentialsjournal.net/labarre.html Consulté le 03/09/18.

20. Labarre, Nicolas, «Appel à contributions : Langage universitaire et bande dessinée - théorie et pratique », Picturing it!, 2016 [en ligne]. Disponible sur : https://picturing.hypotheses.org/422 Consulté le 03/09/18.

21. Bardiaux-Väiente, Marie Gloris et Labarre, Nicolas, Essais. Revue interdisciplinaire d'Humanités, «La bande dessinée, langage pour la recherche », Hors-Série n 5, 2018 [en ligne]. Disponible sur

: https://www.u-bordeaux-montaigne.fr/fr/ecole-doctorale/la-revue-essais/les-numeros-de-larevue/numeros-hors-serie.html Consulté le 13/01/19.

22. Labarre, Nicolas, "Call for Graphic Submissions Special Collection: Graphic Science ", The Comics Grid, 2017 [en ligne]. Disponible sur: https://www.comicsgrid.com/announcement/ Consulté le 03/09/18.

23. Labarre, Nicolas, Métaphores et culture du populaire dans la bande dessinée américaine, Note de synthèse sous la direction de Gabillet, Jean-Paul, Université de Bordeaux Montaigne, 2017, p. 92-123. Le document nous a été aimablement communiqué par l'auteur.

24. En effet, contrairement à la rédaction d'un article sous forme de texte, la bande dessinée académique nécessite de savoir à la fois dessiner de manière intelligible et produire une recherche universitaire, ou alors de former un binôme entre un chercheur et un dessinateur.

25. Sousanis, Nick et Monastersky, Richard, « The Fragile Framework. Can Nations Unite to Save the Earth's Climate? ", Nature, n 527, 2015, p. 427-435.

26. Nick Sousanis a publié dès 2010 des « comics essay » dont on retrouve certaines planches et idées dans sa thèse. Voir par exemple sa participation au numéro en bande dessinée de Visual Art Research : Sousanis, Nick, "The Shape of Our Thoughts : a Meditation on \& in Comics ", Visual Arts Research, "The Graphic Novel and Art Education", Vol. 38, n 1, 2012, p. 1-10. Plusieurs publications de Nick Sousanis, parfois en prépublication, se trouvent sur le site de l'auteur. Voir Sousanis, Nick, « Sousanis Publication List », Spin weave and cut [en ligne]. Disponible sur : http:// spinweaveandcut.com/publication-list/ Consulté le 03/09/18.

27. PROSE signifie « Professional and Scholarly Excellence ».

28. Il existe aussi des traductions en portugais, coréen et serbe.

29. Smolderen, Thierry, « Préface », dans Sousanis, Nick, op. cit., p. 8. L'auteur souligne.

30. Ibid., p. 9. L'auteur souligne.

31. Il est vrai cependant que l'argumentaire de la thèse de $\mathrm{N}$. Sousanis invite précisément à ce type de commentaires.

32. Une étude plus générale des discours universitaires et médiatiques, spécialisés ou non, devrait permettre de dégager une dynamique similaire à ce qui anime le jury du prix Lynd Ward Prize, qui affirme sans détour : "In short, 'Unflattening' takes sequential art to the next level. » «Penn State announces winner of the Lynd Ward Prize for Graphic Novel of the Year », Penn State News, 2016 [en ligne]. Disponible sur : https://news.psu.edu/story/405155/2016/04/19/arts-andentertainment/penn-state-announces-winner-lynd-ward-prize-graphic Consulté le 03/09/18. 
33. Ibid., p. 37.

34. Voir ibid., p. 54 : «Adopter d'autres façons de voir [...] élargit notre monde et révèle des profondeurs insoupçonnées, des espaces plissés appelant l'exploration ».

35. Voir ibid., p. 62 : « Le médium de notre pensée définit ce que nous pouvons voir.» Ibid., p. 69 : «Le visuel fournit l'expression là où les mots échouent ».

36. Ibid., p. 88 : « Dessiner est une manière de voir et ainsi de comprendre, / qui nous fait toucher de plus près les processus perceptions incarnés sous-jacents à la pensée ».

Ibid., p. 90 : «[...] L'activité physique de dessiner est en relation dynamique avec la réaction visuelle de l'artiste à ce qui est couché sur le support ». L'auteur souligne.

37. Le tressage « consiste en une structuration additionnelle et remarquable qui, tenant compte du découpage et de la mise en page, définit des séries à l'intérieur d'une trame séquentielle ». Groensteen, Thierry, Système de la bande dessinée, Paris, Presses universitaires de France, 1999, p. 173.

38. Ibid. Le réseau est aussi évoqué graphiquement à de nombreuses reprises : le labyrinthe, le buisson, la toile d'araignée ou encore les nœuds.

39. Sousanis, Nick, op. cit., p. 77. L'idée est introduite dès le début du chapitre, p. 61 et p. 63 notamment.

40. Ibid., p. 86. Il s'agit d'une citation.

41. Cette forme traverse toute la thèse ; on la retrouve notamment à la p. 140, qui fait aussi l'éloge de la réflexivité.

42. Sousanis, Nick, op. cit., p. 89.

43. À ce titre, il serait intéressant de mettre en regard les travaux de vulgarisation en bande dessinée et ceux relevant de la bande dessinée académique. Il est fort probable que des pratiques et des enjeux puissent être, dans les faits, partagés par l'une et l'autre, volontairement ou non, posant alors, entre autres, la question des publics et des objectifs de la diffusion d'une recherche. Voir par exemple Raichvarg, Daniel et Jacques, Jean, Savants et ignorants: une histoire de la vulgarisation des sciences (1991), Paris, Éditions du Seuil, « Points », 2003, p. 152-163.

44. Sousanis, Nick, op. cit., p. 99.

45. Sousanis, Nick, op. cit., p. 27.

46. D'autres références se trouvent dans ces premières pages, comme cela est précisé pour chaque page où des références sont évoquées, en fin d'ouvrage (voir Sousanis, Nick, op. cit., « Notes », p. 167-179).

47. Ibid., p. 170. Cf. p. 44.

48. Ibid., p. 36.

49. Voir à ce sujet: Thouard, Denis, "La question de la "forme de la philosophie" dans le romantisme allemand », Methodos: savoirs et textes, $\mathrm{n}^{\circ} 1$, La philosophie et ses textes, 2001 [en ligne]. Disponible sur : https://journals.openedition.org/methodos/47 Consulté le 03/09/18.

50. Nancy, Jean-Luc et Lacoue-Labarthe, Philippe, L'Absolu littéraire. Théorie de la littérature du romantisme allemand, Paris, Éditions du Seuil, « Poétique », 1978, p. 22. Les auteurs soulignent. Ici, littérature serait à remplacer par bande dessinée.

51. La «pensée visuelle » est essentiellement étayée par la référence à R. Arnheim qui a publié son livre Visual Thinking en 1969. La psychologie cognitive et les neurosciences ont pourtant depuis lors produit de nombreuses recherches, que nous ne discuterons pas ici, faute de place et d'expertise suffisante. Néanmoins, il faudrait mettre en regard les travaux récents dans ces disciplines et les propositions théoriques de R. Arnheim et de N. Sousanis.

52. Sousanis, Nick, "Behind the Scenes of a Dissertation in Comics Form ", Digital Humanities Quarterly, "Comics as Scholarship ", vol. 9, n 4, 2015 [en ligne]. Disponible sur: http:// www.digitalhumanities.org/dhq/vol/9/4/000234/000234.html Consulté le 03/09/18. Il s'agit de ma traduction de: "[...] through its very form it makes a metaphorical argument for the importance of visual thinking in teaching and learning ». 
53. Nick, Sousanis, op. cit., [en ligne]. Il s'agit de ma traduction de: «I suggest ways to move beyond flatness by engaging in a discussion of interdisciplinarity through the metaphor of perspective - [...] (and again, I never use such terms as "discipline" or "interdisciplinary") ».

54. Par exemple : «Il n'y pas de vue unique, subjective » en terminant la planche par " pour faire de bons penseurs il nous faut de bons voyeurs", Sousanis, Nick, Le Déploiement, op. cit., p. 91. Ou encore : «On ne peut pas plus marcher dans les souliers d'un autre / que voir à travers ses yeux. » Sousanis, Nick, op. cit., p. 99.

55. Par exemple, il introduit les pages 102 et 103 par le cartouche suivant: « Autorisez-moi un exemple personnel».

56. Bouveresse, Jacques, Prodiges et vertiges de l'analogie. De l'abus des belles-lettres dans la pensée, Paris, Raisons d'agir, 1999.

57. Cusset, François, French Theory. Foucault, Derrida, Deleuze et Cie et les mutations de la vie intellectuelle aux États-Unis (2003), Paris, Éditions La Découverte, « La Découverte Poche / Sciences humaines et sociales », 2005, p. 110.

58. Ibid.

59. Bricmont, Jean et Sokal, Alan, Impostures intellectuelles, Paris, Odile Jacob, 1997, p. 14.

60. Il poursuit d'ailleurs avec la métaphore des yeux pour expliquer un calcul d'Ératosthène, appuyé par un schéma scientifique et technique, que T. Smolderen appelle « diagramme » et qu'il présente comme une source de la bande dessinée. Smolderen, Thierry, Naissances de la bande dessinée : de William Hogarth à Winsor McCay, Paris, Les Impressions nouvelles, 2009.

61. Sousanis, Nick, op. cit., p. 42.

62. Cela est vrai également de la French Theory et de ses défenseurs, qui ont largement participé à la diffusion du relativisme cognitif dans les universités d'un côté, mais qui ont aussi assumé un discours politique marqué à gauche (tel que Michel Foucault contre les prisons).

63. Sousanis, Nick, "Behind the Scenes of a Dissertation in Comics Form ", Digital Humanities Quarterly, "Comics as Scholarship », vol. 9, n 4, 2015 [en ligne]. Disponible sur: http:// www.digitalhumanities.org/dhq/vol/9/4/000234/000234.html Consulté le 03/09/18.

Il s'agit de ma traduction de : «[...] the intention of creating a work that doesn't turn the reader away with specialized or politicized language, but can invite them to find their own way of connecting to the material».

64. Sousanis, Nick, op. cit., p. 117. L'auteur souligne.

65. Ibid., p. 141.

66. Ils présentent la critique artiste comme l'indignation contre « d'une part le désenchantement et l'inauthenticité, d'autre part l'oppression, qui caractérisent le monde bourgeois associé à la montée du capitalisme ", tandis que la critique sociale se dresserait contre " l'égoïsme des intérêts particuliers dans la société bourgeoise et la misère croissante des classes populaires dans une société aux richesses sans précèdent [...] » Boltanski, Luc et Chiapello, Ève, Le nouvel esprit du capitalisme (1999), Paris, Gallimard, 2011, p. 88-89.

67. Dubois, Nicole, La norme d'internalité et le libéralisme (1994), Grenoble, Presses universitaires de Grenoble, 2009.

68. Dubois, Nicole, op. cit., p. 158.

69. Sousanis, Nick, op. cit., p. 154.

70. Ibid.

71. Une telle critique sociale de l'université aux États-Unis est menée par exemple par Noam Chomsky. Noam Chomsky, Réflexions sur l'université. Suivies d'un entretien inédit, Ivry-sur-Seine, Raisons d'agir, 2010.

72. Lorsque Sousanis affirme tel ou tel élément, s'agit-il d'une théorie reconnue, d'une étude isolée, d'une métaphore pour expliquer et aider à la mémorisation, d'une opinion ou encore d'une croyance? 
73. Farinella, Matteo, "The Potential of Comics in Science Communication ", Journal of science communication, vol. 17, $\mathrm{n}^{\circ}$ 1, 2018 [en ligne]. Disponible sur : https://jcom.sissa.it/archive/17/01/ JCOM_1701_2018_Y01 Consulté le 03/09/2018.

74. Y. Jablonka, en promouvant les sciences sociales graphiques, le souligne explicitement d'ailleurs : «Ensemble [sciences sociales et arts graphiques], ils détiennent un énorme potentiel cognitif, artistique et, faut-il le dire, commercial. » Voir Jablonka, Yvan, op. cit. [en ligne].

\section{ABSTRACTS}

Cet article s'intéresse à une tendance relativement nouvelle dans l'articulation des savoirs et de la bande dessinée : la production académique dessinée. Nous recensons dans un premier temps plusieurs cas d'articles qui adoptent cette forme, en particulier aux États-Unis mais aussi en France. Nous rendons brièvement compte du succès institutionnel de cette nouveauté et en particulier d'un cas exemplaire : la thèse entièrement dessinée de Nick Sousanis, en sciences de l'éducation. Éditée d'abord par Harvard University Press (2015), elle a été traduite sous le titre Le Déploiement pour Actes Sud - l'An 2 dès 2016.

Afin de prendre du recul sur l'éloge du « langage » que serait la bande dessinée, nous cherchons ensuite à circonscrire les propositions de cette thèse qui légitime sa propre forme afin de proposer une défense et illustration d'une "science" dessinée. Pour l'auteur notre "pensée " serait limitée par le choix d'un médium particulier, ce qui le conduit à utiliser le «langage amphibie » que serait la bande dessinée pour intégrer la pensée visuelle à la recherche. Il critique alors ce qui serait la conception dominante de l'éducation, qui standardiserait l'être humain et limiterait ses potentialités. Ces théories nous conduisent à observer les limites épistémologiques et politiques du Déploiement.

En effet, pour terminer, nous proposons que cette thèse, qui se présente comme une longue métaphore, relève d'un cadre épistémologique peu contraignant, voire d'une adhésion au relativisme cognitif. Fondée en outre sur un abus conceptuel issu de considérations sur la perception, elle peut ainsi être rapprochée du courant postmoderne. Enfin, sur un autre versant, l'ouvrage de N. Sousanis fait la promotion de l'individualisme politique et expose une position libérale tout en refusant un "vocabulaire spécialisé et politisé ». Cet exemple nous amène par conséquent à interroger la nécessité même d'une production académique dessinée qui pourrait traduire, du fait de sa légitimation, le primat de l'esthétique sur la recherche de connaissances en évacuant également, in fine, la critique sociale.

\section{INDEX}

Mots-clés: bande dessinée, légitimité, pédagogie, politique, image, dessin, université 


\section{AUTHOR}

\section{MAXIME HUREAU}

Maxime Hureau est doctorant contractuel depuis septembre 2016 (Laboratoire EHIC - EA 1087 -

Limoges) et a enseigné à ce titre à l'Université de Limoges. Après un Master Texte/Image,

(université de Poitiers/EESI d'Angoulême), dirigé par Désirée Lorenz et Thierry Smolderen, il travaille sur une thèse au sujet de la transmission de savoirs par la bande dessinée nonfictionnelle, sous la direction de Till Kuhnle et Nicole Pignier. 